\title{
Investigating the Depletion Effect: Self-Control does not Waiver in Capuchin Monkeys
}

\author{
Audrey E. Parrish ${ }^{1 *}$, Brielle T. James ${ }^{2,3}$, Mattea S. Rossettie ${ }^{3}$, Travis Smith ${ }^{3}$, \\ Anamaria Otalora-Garcia ${ }^{4}$, and Michael J. Beran ${ }^{2,3}$
}

\author{
${ }^{1}$ Psychology Department, The Citadel, Charleston, SC \\ ${ }^{2}$ Psychology Department, Georgia State University, Atlanta, GA \\ ${ }^{3}$ Language Research Center, Georgia State University, Atlanta, GA \\ ${ }^{4}$ Psychology Department, University of Georgia, Athens, GA \\ *Corresponding author (Email: audrey.parrish1 @ gmail.com)
}

Citation - Parrish, A.E., James, B.T., Rossettie, M.S., Smith, T., Otalora-Garcia, A., \& Beran, M.J. (2018). Investigating the depletion effect: Self-control does not waiver in capuchin monkeys. Animal Behavior and Cognition, 5(1), 118-138. https://doi.org/10.26451/abc.05.01.09.2018

\begin{abstract}
The ego-depletion hypothesis states that self-control diminishes over time and with exertion. However, there is mixed evidence among human adult and comparative studies as to whether such depletion occurs. It is an important issue, given that evidence for or against this hypothesis could have implications for remediation efforts with individuals who show high impulsivity and low self-control. In a study of potential depletion effects on selfcontrol, capuchin monkeys were presented with two consecutive self-control tasks back-to-back within sessions. Monkeys first completed the accumulation task, in which they were presented with food items one-by-one until the subject retrieved and ate the accumulating items, at which point no more food would be delivered. This required continual inhibition of food retrieval in the face of an increasingly desirable reward. Then, monkeys completed a food exchange task with exchange combinations that either decreased or increased in food quality. Self-control was required in foregoing eating an immediately available food for a potentially better reward later in the trial. Individual differences in accumulation performance were observed, but no depletion effects were seen in the monkeys' exchange performance. Next, monkeys were presented with task order counterbalanced across individuals. No order effects were observed in the monkeys' performance on either self-control task. Monkeys' exchange performance was not significantly correlated with accumulation performance in either experiment. These results indicate no depletion effects and that these tasks may not be related in terms of underlying mechanisms that support self-control performance, even though at face value both require inhibition of eating available food.
\end{abstract}

Keywords - Self-control; Ego-depletion; Capuchin monkey; Accumulation task; Food exchange; Delay of gratification

In many circumstances, individuals are faced with a choice between something fairly good now (e.g., a small amount of money or a small treat) or something better later (e.g., a larger amount of money or a larger treat). In these circumstances, self-control is required to forego the immediate reward so as to instead experience a later, but better reward at a future time. Because mishaps of self-control carry steep consequences in today's society (e.g., drug abuse, addiction, gambling, criminal behavior, and social misfortunes), there is an extensive literature devoted to understanding the mechanisms underlying selfcontrol and the factors that lead to its success for some and its failures for others (e.g., Baumeister, 
Heatherton, \& Tice, 1994; Logue, 1988; Mischel, 1974, 2014; Rachlin, 2009; Shoda, Mischel, \& Peake, 1990).

In recent years, a new constraint on self-control choices has been proposed - that decision-makers may suffer greater lapses in their self-control as a function of how often they have to express self-control within a short period of time (i.e., "ego depletion"; Baumeister, Bratslavsky, Muraven, \& Tice, 1998; Baumeister, Vohs, \& Tice, 2007; Baumeister et al., 1994; Muraven, Tice, \& Baumeister, 1998). The classic paradigm used to test the ego-depletion hypothesis presents human subjects with a series of unrelated self-control tasks, the second of which yields lower performance due to resources being depleted in the first task (e.g., Baumeister et al., 1998; Muraven et al., 1998). As one example, Baumeister et al. (1998) presented participants with a desirable food type (cookies and chocolates) and a relatively less-desirable food (radishes) and assigned them to either eat the cookies and chocolates or the radishes. Participants who had to use self-control to stop themselves from eating desirable chocolates and cookies (because they instead had been assigned to eat radishes) gave up on a subsequently presented unsolvable puzzle task faster (i.e., displayed lower self-control) than participants who did not have to exert selfcontrol to resist the tastier chocolate treats (i.e., they were allowed to eat the cookies and chocolates). The idea behind the ego-depletion hypothesis is that self-control may work something like a muscle, in which repeated heavy use can lead to depletion of future strength. Simply put, after having to show self-control in one circumstance, one may not have the capacity (or have decreased capacity) to show it in another circumstance that follows closely thereafter.

The ego-depletion hypothesis has generated a tremendous amount of research, including studies that showed how to offset such depletion (Ackerman, Goldstein, Shapiro, \& Bargh, 2011; Baumeister, Sparks, Stillman, \& Vohs, 2008; Egan, Hirt, \& Karpen, 2011; Job, Walton, Bernecker, \& Dweck, 2013; Muraven, 2010; Schmeichel, Vohs, \& Baumeister, 2003; Vohs et al., 2008). However, there also have been a number of failed efforts to show such self-control depletion, and recent meta-analyses indicate that the depletion hypothesis may not have substantial evidence in support of it (Hagger \& Chatzisarantis, 2017; Hagger, Wood, Stiff, \& Chatzisarantis, 2010; Vadillo, Gold, \& Osman, 2016). Thus, it remains unclear how best to conceptualize the role that performing one self-control task might have on subsequent tasks that also require degrees of inhibition and self-control (e.g., Carter \& McCullough, 2014; Inzlicht \& Schmeichel, 2012; Inzlicht, Schmeichel, \& Macrae, 2014).

The history of studying self-control in psychological experiments has always had a strong comparative perspective. This is particularly true for research using intertemporal choice tasks, in which subjects make a discrete smaller-sooner versus larger-later choice (e.g., Ainslie, 1974; Berns, Laibson, Loewenstein, 2007; Deluty, 1978; Green, Myerson, Holt, Slevin, \& Estle, 2004; Logue, 1988; Logue \& Chavarro, 1987; Navarick \& Fantino, 1976; Rachlin \& Green, 1972; Stevens, Hallinan, \& Hauser, 2005; Stevens \& Mühlhoff, 2012; Tobin, Chelonis, \& Logue, 1993; van Haaren, van Hest, \& van de Poll, 1988). However, nonhuman animals also have performed a variety of other tasks, including delay of gratification tasks (e.g., Anderson, Kuroshima, \& Fujita, 2010; Beran, 2002; Beran \& Evans, 2006; Beran, SavageRumbaugh, Pate, \& Rumbaugh, 1999; Brucks, Soliani, Range, \& Marshall-Pescini, 2017; Evans \& Beran, 2007; Evans, Beran, Paglieri, \& Addessi, 2012; Grosch \& Neuringer, 1981; Hillemann, Bugnyar, Kotrschal, \& Wascher, 2014; Koepke, Gray, \& Pepperberg, 2015; Parrish et al., 2014; Stevens, Rosati, Heilbronner, \& Mühlhoff, 2011), food exchange tasks (Beran, Rossettie, \& Parrish, 2016; Dufour, Pelé, Sterck, \& Thierry, 2007; Pelé, Dufour, Micheletta, \& Thierry, 2010; Pelé, Micheletta, Uhlrich, Thierry, \& Dufour, 2011; Ramseyer, Pelé, Dufour, Chauvin, \& Thierry, 2006), token exchange tasks (e.g., Beran \& Evans, 2012; Bourjade, Thierry, Call, \& Dufour, 2012; Hackenberg \& Vaidya, 2003; Judge \& Essler, 2013; Parrish, Evans, Perdue, \& Beran, 2013), and other tasks in which a more immediately available reward has to be avoided to gain a better reward that is delayed (Blanchard \& Hayden, 2015; Bramlett, Perdue, Evans, \& Beran, 2012; Evans \& Westergaard, 2006; Mayack \& Naug, 2015). Thus, with such varied tasks to choose among, it seems likely that comparative data also can be informative to the literature on depletion.

To date, there have been limited attempts to look at self-control depletion in other species. Some reports have emerged suggesting that dogs may show a depletion-like effect (e.g., Miller, 2013; Miller \& 
Bender, 2012; Miller, DeWall, Pattison, Molet, \& Zentall, 2012; Miller, Pattison, DeWall, RayburnReeves, \& Zentall, 2010). For example, Miller et al. (2010) tested domestic dogs (Canis familaris) on a consecutive task paradigm similar to the Baumeister et al. (1998) cookie-radish study. Dogs in the selfcontrol condition were required to stay in the 'sit' position for a total of 10 minutes, whereas dogs in the control condition were placed in a dog cage for the same amount of time. Dogs tested in the self-control condition persisted for significantly less time on an unsolvable task (manipulating a toy with an unattainable treat) than dogs tested in the control condition, suggesting human-like depletion effects using a consecutive task paradigm. However, research with nonhuman primates has shown little evidence for depletion. For example, De Petrillo and colleagues assessed depletion effects in capuchin monkeys (De Petrillo et al., 2015). Monkeys performed an accumulation task in which items accrued in front of them, and the monkeys had to not eat the items in order to keep the accumulation of rewards going. De Petrillo et al. compared performance on that task when monkeys were tested before receiving their daily meal or after receiving it. They also examined accumulation performance after being tested in two tasks that varied in their cognitive complexity. Accumulation performance decreased within-session across trials, but there was no effect as a function of when the meal was eaten or the difficulty of the previously completed cognitive task. Thus, there was some evidence of depletion but not as a function of meal consumption or prior task exposure as has been documented in dogs and adults using a consecutive task paradigm.

Parrish, Emerson, Rossettie, and Beran (2016) also assessed in capuchin monkeys a corollary to the ego-depletion hypothesis that involves a potential role of glucose in self-control. This glucose hypothesis states that depletion may occur because of diminished glucose available to the subject, and that a boost in glucose would essentially reinstate previously-exerted self-control resources (e.g., Gailliott \& Baumeister, 2007; Sanders, Shirk, Burgin, \& Martin, 2012). After an overnight period in which no food was available to monkeys, Parrish et al. gave those monkeys a breakfast meal high in glucose or nearly devoid of glucose, and then assessed self-control performance in the accumulation task. There was no effect of glucose ingestion levels on performance during the accumulation task, a finding that matched that of some glucose studies with humans (e.g., Lange \& Eggert, 2014). Thus, to date it appears that the lack of depletion effects in capuchin monkeys may be a "meaningful failure" to demonstrate comparative similarities with some results reported in humans. However, with such limited attempts, it is necessary first to collect more data before drawing any strong conclusions.

In the current study, capuchin monkeys were presented with two consecutive self-control tasks. We chose capuchin monkeys for our previous test of the glucose hypothesis (Parrish et al., 2016) and the current study as, aside from chimpanzees, they are the most widely utilized primate model for self-control research (e.g., Addessi \& Rossi, 2011; Addessi et al., 2013; Anderson et al., 2010; Beran et al., 2016; Bramlett et al., 2012; Evans et al., 2012; Evans, Perdue, Parrish, \& Beran, 2014; Paglieri et al., 2013; Pelé et al., 2011), including the recent assessment of depletion effects (De Petrillo et al., 2015). Of relevance to the current work, there is individual variability in capuchin monkey performance in selfcontrol tasks including the accumulation test (e.g., Evans et al., 2012; Parrish et al., 2016), which may lead to interesting differences in the depletion effect as studied here. In the present study, we varied the parameters of each task, including task order, to investigate if depletion effects emerged within capuchin monkeys and, if so, to assess the factors affecting such depletion. We included two self-control tasks that are well known in the comparative literature and commonly used for assessing self-control in primates. The first task was the accumulation task in which food items were presented one at a time until the animal retrieved and consumed the items (Anderson et al., 2010; Beran, 2002; Beran \& Evans, 2006; Evans \& Beran, 2007; Parrish et al., 2014; Stevens et al., 2011; Vick, Bovet, \& Anderson, 2010). The dependent measure of self-control was the number of items that an animal accumulated before consuming a food item. Crucial to the current task design, we varied whether monkeys were required to use self-control to inhibit consuming the items in the accumulation process. To do so, we included a free accumulation condition in which the food accumulated in reach of the monkey (thus requiring self-control) and a forced accumulation condition in which the food accumulated out of reach of the monkey (thus not requiring self-control). This variance in food accessibility allowed us to design scenarios in which a second self- 
control task (described below) followed a situation in which self-control should be depleted (following free accumulation) versus a nearly identical situation in which self-control should not be depleted (following forced accumulation) but an animal still had to wait through a delay interval to eat food items. This design allowed for control over several variables that may impact depletion effects but that do not relate to depletion itself, including caloric intake, presence of prepotent stimuli, time between tasks, and fatigue effects.

The second task used in the current study was a food exchange task in which monkeys were presented with different food items that varied in their relative quality or preference ranking, with the objective to exchange the food items with the experimenter until the best available item was received. This, too, is a commonly used task to assess delay of gratification in nonhuman species (e.g., Beran et al., 2016; Dufour et al. 2007; Judge \& Essler, 2013; Pelé et al., 2010; Pelé et al., 2011; Ramseyer et al., 2006). Exchange tasks are particularly suited to test the ego-depletion hypothesis because they require continued exertion of self-control in terms of actively engaging in trading behavior to obtain the highest valued item. The dependent measure of self-control for this task is the item that is eaten and its preference ranking relative to the other available foods. For example, an optimal response would be to exchange a lower-valued item (e.g., a carrot slice) for a higher-valued item (e.g., an apple piece or a marshmallow). Self-control is required in foregoing the immediately available food item (the carrot) for a potentially better reward (the marshmallow) that the monkey would receive if it exchanged the first item. Previous comparative assessments have required self-control exertion via tasks that require waiting (e.g., stay in the 'sit' position for 10 minutes) as the first test to deplete self-control resources (e.g., Miller et al., 2010) or used the same task across multiple trials within a session (De Petrillo et al., 2015). We felt that the choice of two different active-type self-control tasks would provide the best possible opportunity to assess depletion effects in capuchin monkeys.

Our second objective for the current work was to assess whether performance across these two self-control tasks correlated with one another within individuals. There is a growing interest in establishing self-control assessment batteries that contain a variety of behavioral inhibition or self-control measures (e.g., Brucks, Marchall-Pescini, Wallis, Huber, \& Range, 2017; MacLean et al., 2014; see Beran, 2015, for a review). MacLean et al. (2014) recently assessed 567 animals (representing 36 different species) on two inhibitory control paradigms (an A-not-B task and the cylinder/barrier task) and reported that absolute brain volume (but not social group size) predicted performance on these tests of inhibition. Other research also has indicated that, although at face value certain tasks would seem to rely on similar inhibitory mechanisms, performance across tasks does not appear to be as closely related as might be expected, in humans (e.g., Reynolds \& Schiffbauer, 2005) and in nonhumans (e.g., Addessi et al., 2013; Brucks et al., 2017a). To assess whether the tasks used in the current study (accumulation and food exchange) were related to one another within the same subject, we correlated monkeys' performance across the two tasks.

Considering the variability in human and nonhuman animal studies concerning the depletion hypothesis, we did not have directional hypotheses regarding the effects of consecutive tasks on selfcontrol performance. If capuchin monkeys were susceptible to depletion effects, we should see a decline in self-control performance in the second task (food exchange) following a task requiring the animals to use self-control (free accumulation) relative to a task that did not require animals to use self-control (forced accumulation). Further, if depletion effects were to occur, we should see that the order in which tasks were presented would not factor into such depletion effects, given that the second task in any sequential presentation should be impacted negatively. Finally, if we found a significant correlation between the two self-control tasks, it would indicate that both tasks might tap into similar behavioral mechanisms in this species. 


\section{Experiment 1}

\section{Method}

Subjects. Ten adult capuchin monkeys were included in the following experiments, six females (Gambit, Lexi, Lily, Nala, Widget, and Wren: ages 8 to 19 years) and four males (Gabe, Griffin, Liam, and Logan: ages 11 to 18 years). One female (Lexi) would not eat a subset of the selected rewards (minimarshmallows), and thus was dropped after food preference testing. Another female (Gambit) would not readily engage in testing, and thus was dropped from the study after exchange training. Their data are not included in the Results. Monkeys were group-housed at Georgia State University's Language Research Center with conspecifics in indoor/outdoor enclosures and supplied with enrichment, including climbing structures, toys, browse, and other forms of enrichment. Monkeys voluntarily separated for solo testing during which they had visual and auditory access to group mates. They received a daily diet of chow, fruits, and vegetables, and were never food deprived or weight reduced for testing purposes. Water was available ad libitum. Testing complied with the procedures and protocols that were approved by the Institutional Animal Care and Use Committee of Georgia State University (GSU). GSU is accredited by the Association for Assessment and Accreditation of Laboratory Animal Care. These monkeys had participated in several self-control studies (Beran et al., 2016; Bramlett et al., 2012; Evans \& Beran, 2014; Evans et al., 2014), including previous studies assessing self-control performance on the accumulation task (Addessi et al., 2013; Evans et al., 2012, Paglieri et al., 2013; Parrish et al., 2016).

Apparatus. For all test sessions, monkeys voluntarily entered individual stainless steel mesh test boxes $(33 \times 46 \times 61 \mathrm{~cm})$ that were attached to the group enclosure. Experimenters could exchange food items with the monkeys via the side of the stainless steel mesh test boxes, offering items to the monkey or receiving items offered by the monkey through the steel mesh. A vertical Plexiglas panel was attached to one end of the test enclosure. A hinged Plexiglas pan $(15 \times 7.5 \mathrm{~cm})$ was attached to the front of the panel so that its contents were accessible to the monkey and the experimenter (if the pan was hinged inwards; see Figure 1A) or to the experimenter only (if the pan was hinged outwards; see Figure 1B). A deadbolt lock could be used to lock the pan in the outwards position so that only the experimenter could access its contents.

A)

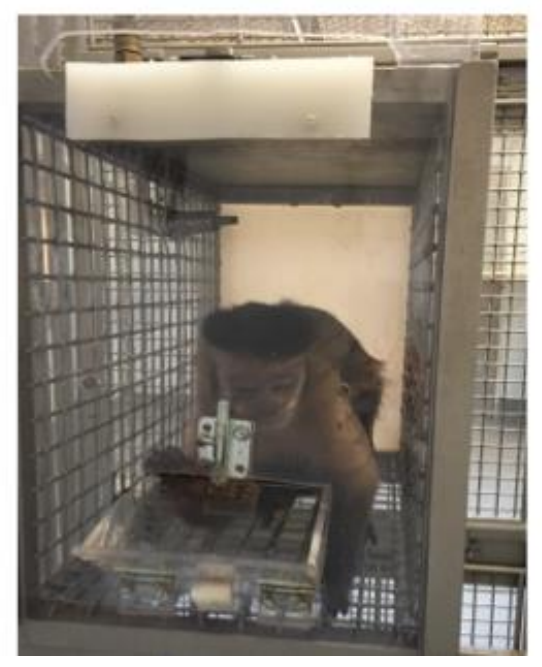

B)

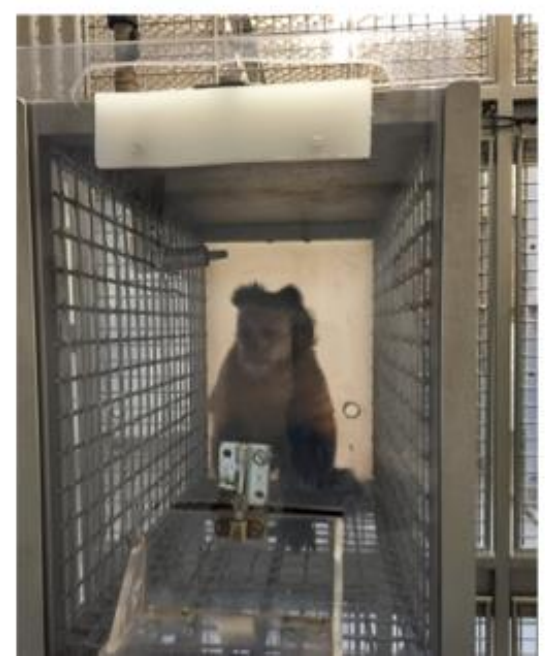

Figure 1 (A) Self-control accumulation task set-up. Food pan unlocked so monkey and experimenter can access its contents (used in Free Accumulation condition). B) Self-control accumulation task set-up. Food pan locked so monkey cannot access its contents until experimenter unlocks pan (used in Forced Accumulation condition). Reprint from Parrish et al., 2016. 


\section{General Procedure}

Food preference testing. We first conducted food preference tests to establish different valued food items to be included in the food exchange task (described below). We selected a high-valued reward (one mini-marshmallow), a medium-valued reward ( $2 \mathrm{~g}$ apple piece), and a low-valued reward ( $2.5 \mathrm{~g}$ carrot slice). Monkeys were presented with pairwise comparisons of each food type (mini-marshmallow vs. apple piece, mini-marshmallow vs. carrot slice, apple piece vs. carrot slice) to assess relative preferences between the different foods.

Trials were presented via a rolling cart with a tray $(45.5 \times 68.5 \mathrm{~cm})$ attached to the top thas positioned directly in front of the monkey's test box. The Plexiglas panel attached to the front of the test box had a circular opening $(4.5 \mathrm{~cm}$ in diameter) through which the monkey could reach its arm and easily make a selection between the items presented on the cart. The two food items for a given trial were placed on the tray approximately $15 \mathrm{~cm}$ apart and food placement (right/left) was randomized across trials. After the tray was baited, the cart was pushed forward towards the monkey who then made a selection of one of the two food types by reaching through the armhole and retrieving the preferred item. The monkey consumed the selected reward immediately and the experimenter removed the unselected item from the tray. Total trial length was $30 \mathrm{~s}$, which began when the tray was pushed forward to the monkey. Following the $30 \mathrm{~s}$ interval, the cart was moved backwards toward the experimenter who baited the tray for the next trial.

Monkeys received two food preference test sessions on different days, with six trials per session (for a total of 12 trials). In each session, two trials of each pairwise comparison were presented. All monkeys selected the higher-valued food in $100 \%$ of trials (marshmallow > apple, marshmallow > carrot, and apple > carrot). It is important to note that the monkeys were all willing to eat the lower-valued food item and did so on a regular basis as part of their routine diets; thus, we selected two lower-preference, but still desirable, items that the monkeys were known to eat frequently.

\section{Training on Self-Control Tasks}

Food exchange training. Monkeys were trained to complete a food item exchange procedure in which they were offered one food item by the experimenter that they could either consume immediately or exchange for another item offered by the experimenter. Self-control was required in that the monkeys needed to inhibit eating the first item in order to exchange it for a potentially more desirable option.

Monkeys were seated in their individual testing box (as described above) and were first shown one of the three food items that would be available for exchange (mini marshmallow, apple piece, or carrot slice) at a later time by the first experimenter (E1). E1 then closed his/her eyes and placed one hand palm up in front of the monkey via the small circular opening in the front of the Plexiglas window in a begging gesture. E1's second hand was positioned directly behind his/her first hand and contained the item that was available for exchange (this food item was out of reach of the monkey but fully visible). The second experimenter (E2) then gave the monkey one of the other two remaining food items via the side mesh of the testing box. The monkey could either (a) consume the first food item received from E2 or (b) exchange that food item with E1 by placing the first food item on E1's open palm. Depending upon the behavior of the monkey, E2 announced "eat" if the monkey consumed the first item or "exchange" if the monkey placed the first item on E1's palm. If the monkey exchanged the first food item, E1 gave the monkey the item in his/her other hand for consumption. To control for cuing, E1 did not know which food item was first given to the monkey by E2 and, thus, was blind to whether the monkey should exchange or should not exchange the food based on its relative value to the second item. Total trial length was $30 \mathrm{~s}$, regardless of whether the monkey consumed the first food or exchanged the food for the second item.

A total of eight exchange trial types were included (see Table 1). These included four Quality Decreasing trials (A - one mini-marshmallow for carrot slice, B - apple piece for carrot slice, C - one mini-marshmallow for apple piece, D - carrot slice for nothing/no food). The optimal response for Quality 
Decreasing trials was to keep and consume the higher-valued item received first and to refrain from exchanging the item for the lower-valued outcome (e.g., do not exchange a mini-marshmallow for a carrot slice). We included Quality Decreasing trial types to ensure that the monkeys did not 'blindly' exchange all items received, but instead made exchanges only when those exchanges led to a higher-valued food item. We also presented four Quality Increasing trials (A - carrot slice for one mini-marshmallow, B carrot slice for apple piece, C - apple piece for one mini-marshmallow, D - one mini-marshmallow for three mini-marshmallows). The optimal response for Quality Increasing trials was to inhibit eating the lower preference item received first and to exchange the item for the higher-valued food (e.g., exchange a carrot slice for a mini-marshmallow).

Table 1

Food Exchange Trial Type Descriptions for Exchange Training in Experiment 1

\begin{tabular}{lccc}
\hline \multicolumn{1}{c}{ Trial Type } & First Food Item & Item For Exchange & Optimal Response \\
\hline Quality Decreasing A & 1 Mini-Marshmallow & Carrot Slice & Eat $1^{\text {st }}$ item \\
Quality Decreasing B* & Apple Piece & Carrot Slice & Eat $1^{\text {st }}$ item \\
Quality Decreasing C & 1 Mini-Marshmallow & Apple Piece & Eat $1^{\text {st }}$ item \\
Quality Decreasing D & Carrot Slice & Nothing/No food & Eat $1^{\text {st }}$ item \\
\hline Quality Increasing A* & Carrot Slice & 1 Mini-Marshmallow & Exchange $1^{\text {st }}$ item \\
Quality Increasing B* & Carrot Slice & Apple Piece & Exchange $1^{\text {st }}$ item \\
Quality Increasing C* & Apple Piece & 1 Mini-Marshmallow & Exchange $1^{\text {st }}$ item \\
Quality Increasing D & 1 Mini-Marshmallow & 3 Mini-Marshmallows & Exchange $1^{\text {st }}$ item \\
\hline
\end{tabular}

*These four trial types were included in exchange testing for Experiment 1.

Each monkey completed 10 training sessions administered on different days, with one of the eight trial types described above per session (for a total of 80 trials). We recorded which of the two items the animals ate (first item - no exchange or second item - exchange), and we did not allow the animals to make an exchange if they partially consumed the first piece of food.

Accumulation training. Next, monkeys completed a session of the accumulation task in which they were re-introduced to the parameters of the paradigm. Because all monkeys had prior experience with this task (Addessi et al., 2013; Beran et al., 2016; Evans et al., 2012, Paglieri et al., 2013; Parrish et al., 2016), they were not exposed to a longer training routine as described above for the food exchange task. In the forced-accumulation trials, the pan attached to the front of the Plexiglas test box plate was locked in the outwards position so that the monkeys could not access its contents until the experimenter unlocked the pan (see Figure 1B). In this trial, the experimenter transferred 10 pellets (94 mg bananaflavored pellets from Bio-Serv, Frenchtown, NJ) into the pan from a transparent bowl at a 2-s delivery rate for each pellet. The experimenter then unlocked the pan, which gave monkeys access to the accumulated rewards. These forced-accumulation trials were used to remind the monkeys of the accumulation procedure, but did not require self-control as the contents were not accessible until the experimenter unlocked the pan after all items were transferred.

In the free-accumulation trials, the pan deadbolt was left unlocked so that the monkey could access the pan's contents at any time. Thus, to obtain more items, monkeys had to inhibit retrieving and consuming the accumulating items. The experimenter transferred up to 20 pellets (one at a time) from a transparent bowl into the pan or until the monkey consumed a pellet, which ended the accumulation period. Rewards again were transferred at a 2-s delivery rate. Total trial length for the forced accumulation trials was $30 \mathrm{~s}$ and total trial length for the free accumulation trials was $90 \mathrm{~s}$. Following completion of the trial duration, the next trial immediately began.

Each monkey completed one training session on a separate day from testing (described below). Each training session presented two forced accumulation trials and two free accumulation trials as described above, alternating between forced and free trials (one forced trial with 10 pellets, one free trial with 20 pellets, one forced trial with 10 pellets, and one free trial with 20 pellets). We recorded the total number of pellets accumulated in all sessions. 


\section{Testing}

Accumulation testing. To test for any self-control depletion effects, capuchin monkeys completed the accumulation self-control task followed immediately by the food exchange self-control task. Crucial to the task design, the free accumulation condition was compared to the forced accumulation condition in which the monkeys were not required to inhibit taking the accumulating food items. The prediction was that if depletion occurred, the monkeys' exchange performance should suffer following the free accumulation condition (which required self-control because the pan's contents were freely accessible throughout the trial, possibly depleting self-control resources) to a greater degree than their exchange performance following the forced accumulation condition (which did not require self-control, although it did require waiting through a delay until food was delivered).

In the free accumulation condition, the experimenter transferred up to 50 pellets (one at a time) from a transparent bowl into the unlocked and accessible pan until the monkey consumed a reward, which ended the accumulation period. Rewards were transferred at a 2-s delivery rate. In the forced accumulation condition, the pan was locked in the outwards direction as described above for Training. The number of pellets received by the monkey in the forced condition was yoked to the number of pellets received by the monkey on the previous free accumulation testing day. This allowed for the control of caloric intake prior to the exchange task to ensure that any observed differences in exchange behavior between free and forced accumulation days was not a factor of satiation or the length of delay, but were the result of a difference in terms of the self-imposed inhibition exhibited in the preceding accumulation trial. In the forced accumulation trials, pellets were transferred into a transparent bowl on top of the tray positioned in front of the testing box. Once the experimenter transferred the total number of pellets to the bowl, he or she then delivered these pellets into the pan, which was unlocked at that time and the pellets became accessible to the monkey. We chose to transfer the pellets into a visible bowl (rather than directly into the pan) to reduce any attempts by the monkeys to open the locked pan. For all accumulation testing sessions, we recorded the total number of items obtained. The exchange session began $60 \mathrm{~s}$ after the completion of the preceding accumulation session.

Food exchange testing. Food exchange trials were identical to those described in Training, except that we used only one experimenter for the exchange trials. Now, E1 administered the accumulation session and then E2 administered the exchange session. Importantly, E2 did not know whether the monkeys were in a free or forced accumulation condition as he/she was out of the testing room for the initial task. This eliminated the possibility of E2 cuing the animals to exchange more or less as a function of the first task (forced vs. free accumulation).

A total of four exchange trial types were included. These included three Quality Increasing trials (A - carrot slice for one mini-marshmallow, B - carrot slice for apple piece, C - apple piece for one minimarshmallow) and one Quality Decreasing trial (A - apple piece for carrot slice). Because during training the monkeys had rarely exchanged one mini-marshmallow for three mini-marshmallows or a minimarshmallow for either of the other food types, we eliminated these trials. Exchange trials were $30 \mathrm{~s}$ in length, regardless of exchange behavior.

Each monkey completed 10 test sessions administered on different days, with an accumulation phase (free or forced) followed by an exchange phase in each session. The exchange phase included eight total trials, with two trials of each trial type described above (presented in random order). Each monkey completed five sessions with free accumulation and five sessions with forced accumulation. Free and forced accumulation sessions were presented in two-session blocks (on different days), with a free session followed by a forced session on the following day. 


\section{Results}

\section{Training}

Food exchange training. We first considered performance in the food exchange task by splitting the trial types into the Quality Decreasing trials (optimal response was to eat the first higher-valued food item rather than exchanging it for the second lower-valued food item) and the Quality Increasing trials (optimal response was to exchange the first lower-valued food item for the second higher-valued food item). These data are plotted as a function of optimal performance (eat or exchange) for the eight trial types (see Figure 2). Using a Fisher's Exact test to compare exchange performance, we found that six of the eight monkeys were significantly more likely to exchange the food item in the Quality Increasing trials than the Quality Decreasing trials, including Gabe $(p=.004)$, Griffin $(p=.006)$, Lily $(p<.001)$, Logan $(p<.001)$, Nala $(p=.02)$, and Wren $(p=.002)$. Liam $(p=.06)$ displayed performance patterns in the predicted manner. Widget $(p=.62)$ rarely exchanged in either the Quality Decreasing trial types $(1 / 40$ trial) or Quality Increasing trial types (3/40 trials). These results suggested that the majority of monkeys learned to exchange the first item when the trade could lead to a better food reward (Quality Increasing trials) and did not exchange the first item when the trade would lead to a less-valued food reward (Quality Decreasing trials). Based upon a visual examination of the data, we also noted that the monkeys varied in their ability to exchange for the better item in Quality Increasing trials when the first item was of medium value (apple-to-marshmallow) and no monkeys completed the quantitative exchange of 1 marshmallowto-3 marshmallows. This is a point to which we return in the test results below.

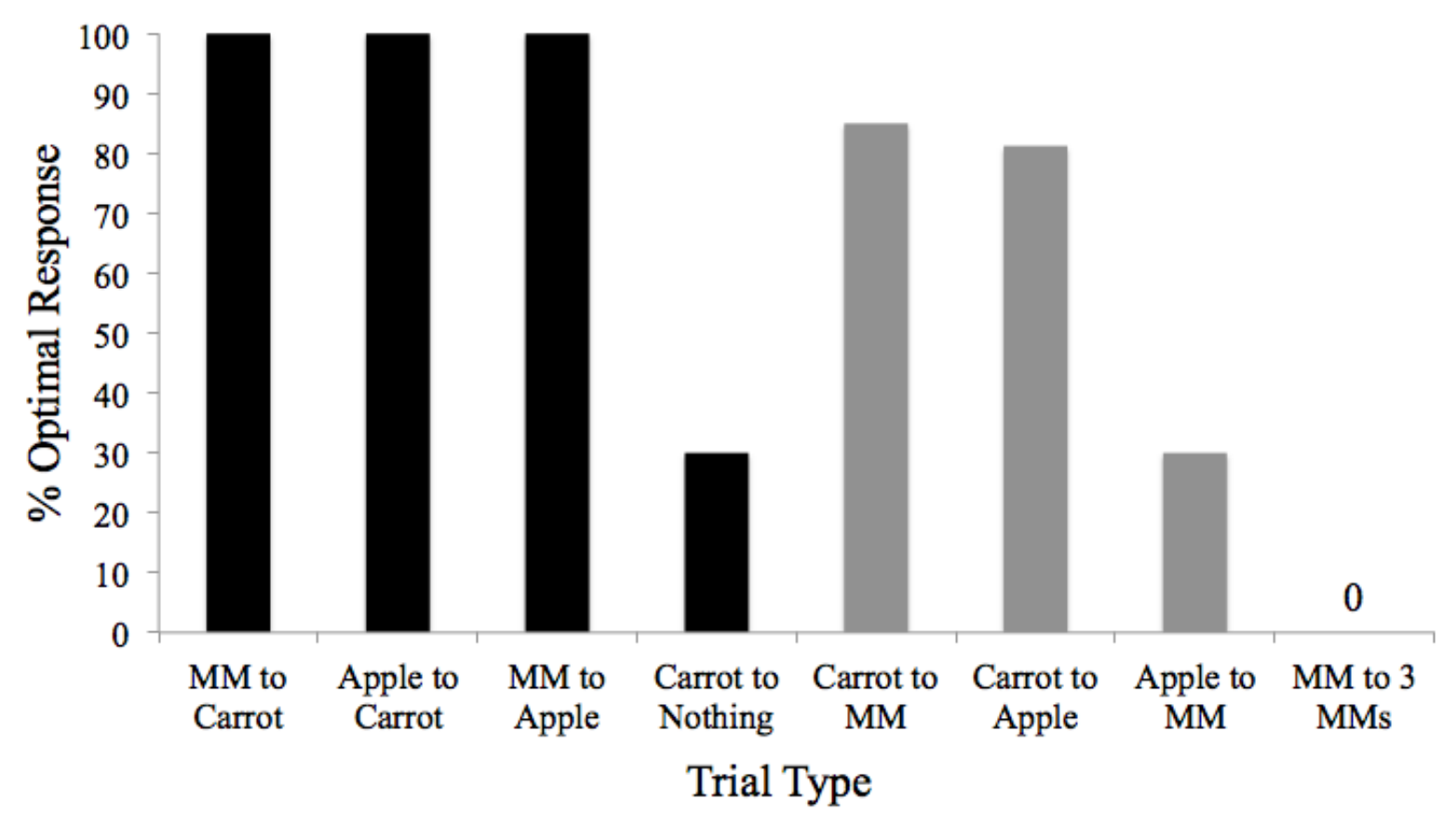

Figure 2. Training performance for the food exchange self-control task in Experiment 1. Black bars depict Quality Decreasing trials (optimal response was to eat the first higher-valued food item rather than exchanging it for the second lower-valued food item). Gray bars depict Quality Increasing trials (optimal response was to exchange the first lower-valued food item for the second higher-valued food item). Note that MM represents one mini-marshmallow. 
Accumulation training. There was variability in the monkeys' performance during the accumulation training, which was expected given the results of previous accumulation studies (e.g., Parrish et al., 2016). The total number of pellets (maximum: 20 pellets) accumulated in the two free accumulation trials is as follows: Gabe (10 and 12), Griffin (10 and 10), Liam (11 and 18), Lily (20 and 20), Logan (20 and 20), Nala (8 and 15), Widget (1 and 1), and Wren (20 and 20). Our goal was not to train monkeys to accumulate all available items, but to give them some reminder experience with the task and with how the apparatus worked (unlocked pan in free accumulation trials and locked pan in forced accumulation trials).

\section{Testing}

Accumulation testing. Table 2 presents the total number of pellets accumulated for each monkey across the five free accumulation test days. These data are identical for the five forced accumulation test days, given that the forced pellet number was yoked to the previous free accumulation day. The average number of pellets accumulated across the five free sessions (maximum: 50 pellets per session) for each monkey is as follows: Gabe $(M=14.6)$, Griffin $(M=15.4)$, Liam $(M=22.8)$, Lily $(M=37.6), \operatorname{Logan}(M$ $=33.2)$, Nala $(M=4.8)$, Widget $(M=1.0)$, and Wren $(M=19.6)$. As previous accumulation testing has shown (e.g., Beran et al., 2016; Parrish et al., 2016), there was high variability in accumulation test performance by individual monkeys, with some monkeys accumulating almost all 50 pellets in each session (Lily) whereas other monkeys accumulated only between one and six pellets for all sessions (Widget and Nala).

Table 2

Accumulation Test Performance from Experiment 1 (Total Number of Pellets Accumulated; Maximum Possible: 50 Pellets) for the Five Free Accumulation Sessions

\begin{tabular}{ccccccccc}
\hline Session \# & Gabe & Griffin & Liam & Lily & Logan & Nala & Widget & Wren \\
\hline $\mathbf{1}$ & 13 & 20 & 31 & 50 & 34 & 4 & 1 & 28 \\
$\mathbf{2}$ & 16 & 10 & 26 & 22 & 2 & 6 & 1 & 27 \\
$\mathbf{3}$ & 16 & 13 & 25 & 16 & 41 & 6 & 1 & 18 \\
$\mathbf{4}$ & 13 & 19 & 13 & 50 & 39 & 4 & 1 & 16 \\
$\mathbf{5}$ & 15 & 15 & 19 & 50 & 50 & 4 & 1 & 9 \\
Average & 14.6 & 15.4 & 22.8 & 37.6 & 33.2 & 4.8 & 1 & 19.6 \\
\hline
\end{tabular}

Food exchange testing. Figure 3 depicts individual monkey performance in the four exchange trials as a function of accumulation condition (free vs. forced). Optimal performance for the Quality Decreasing trial type (apple-to-carrot) was to consume the higher-valued first food item (no exchange), whereas optimal performance for the three Quality Increasing trial types was to exchange the lowervalued first item for the higher-valued second item. There were individual differences in exchange performance across trial types, but importantly, there was no significant difference in optimal exchange performance behavior following the two accumulation conditions (Fisher's Exact Test; all $p \mathrm{~s}>.05$ ). Thus, engaging in a self-control task that potentially depleted resources (free accumulation) had no effect on a secondary self-control task (food exchange) above the effect of a nearly identical task that did not require inhibition (forced accumulation). 
(A) Quality Decreasing (Apple to Carrot)

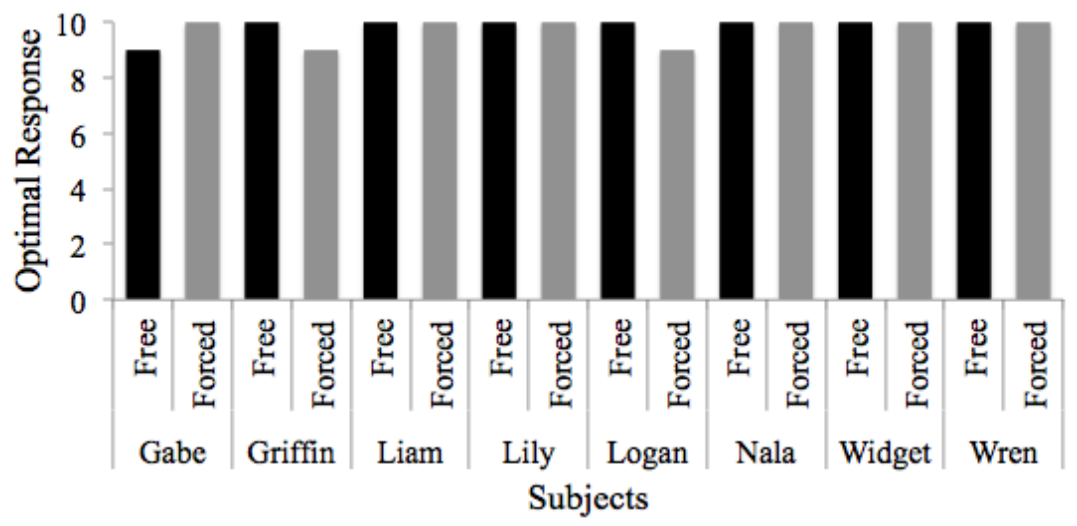

(C) Quality Increasing (Carrot to Apple)

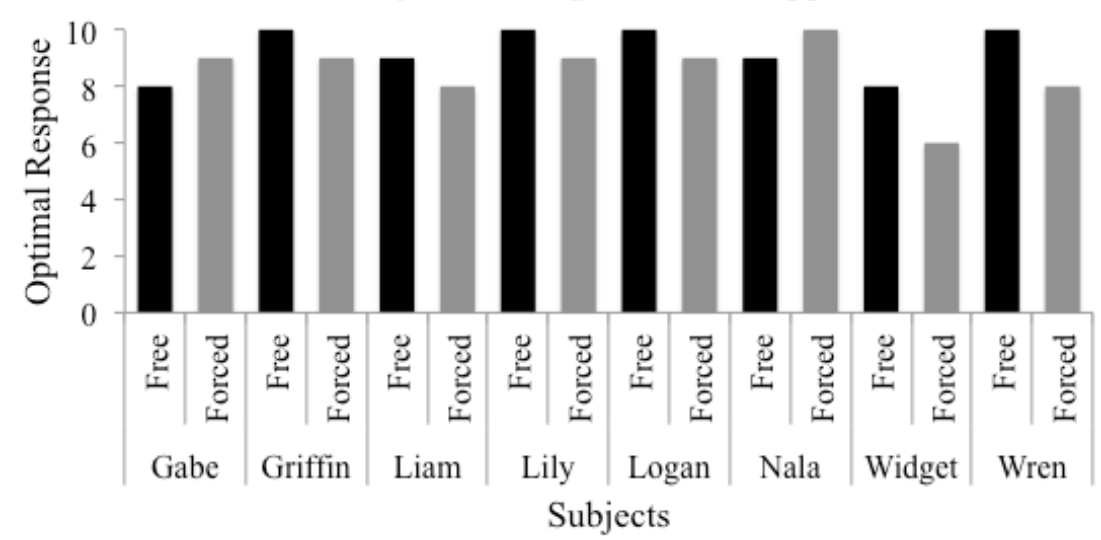

(B) Quality Increasing (Carrot to Marshmallow)

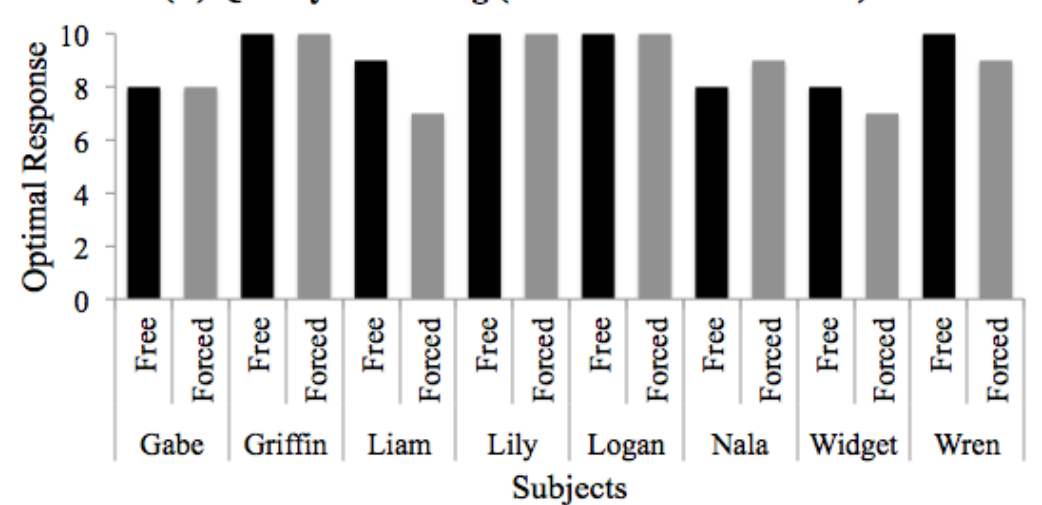

(D) Quality Increasing (Apple to Marshmallow)

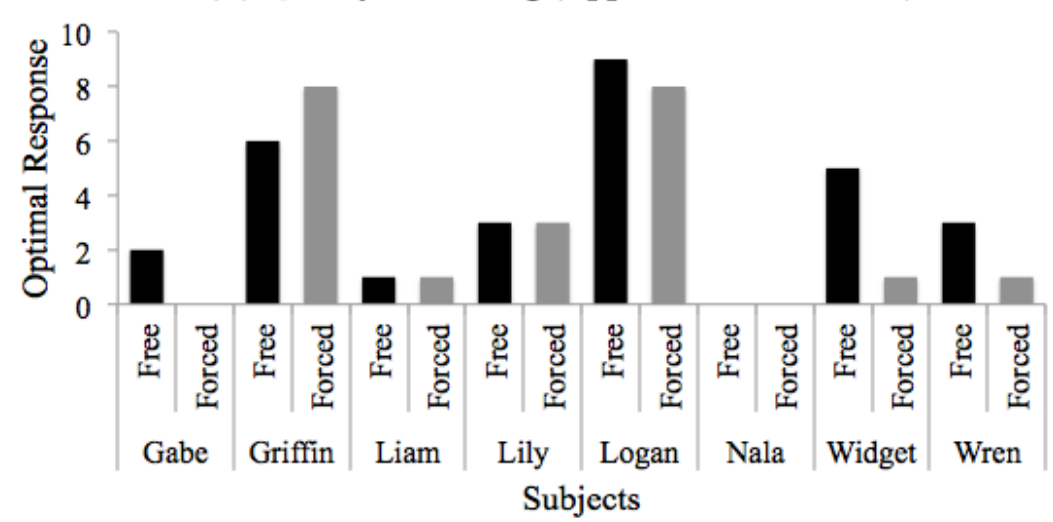

Figure 3. Test performance for the food exchange self-control task in Experiment 1, including the (A) Quality Decreasing trial type - Apple-to-Carrot, (B) Quality Increasing trial type - Carrot-to-Marshmallow, (C) Quality Increasing trial type - Carrot-to-Apple, and (D) Quality Increasing trial type - Apple-to-Marshmallow. 
Furthermore, we were interested in whether there were differences in optimal performance across the different exchange trial types. There were four trial types: apple-to-carrot, carrot-to-marshmallow, carrotto-apple, and apple-to-marshmallow. Collapsing across free and forced conditions, we conducted a oneway repeated measures ANOVA to compare optimal performance across the four exchange trial types. Because of a violation of the assumption of sphericity, we used the Greenhouse-Geisser correction $($ epsilon $=.402)$ for this ANOVA. This analysis revealed a significant difference among these trial types for capuchin monkeys, $F(1.21,8.43)=32.50, p<.001$, partial $\eta^{2}=.823$. To examine this effect more closely, we compared performance for select comparisons to evaluate the role of relative food value, using a Bonferroni correction with alpha set at .0167 (.05/3). Consistent with training results, a visual inspection of the data in Figure 4 revealed that monkeys exchanged less often in the apple-tomarshmallow trial type $(M=31.88 \%, S D=30.23 \%)$, in which they needed to inhibit eating a mediumvalued food (apple) in exchange for a higher-valued food (marshmallow). Optimal performance was significantly lower for this trial type relative to all other trial types, apple-to-carrot $(M=98.13 \%, S D=$ $2.59 \%), t(7)=-5.87, p=.001$, carrot-to-marshmallow $(M=89.38 \%, S D=10.50 \%), t(7)=-6.60, p<.001$, and carrot-to-apple $(M=88.75 \%, S D=8.76 \%), t(7)=-5.57, p=.001$.

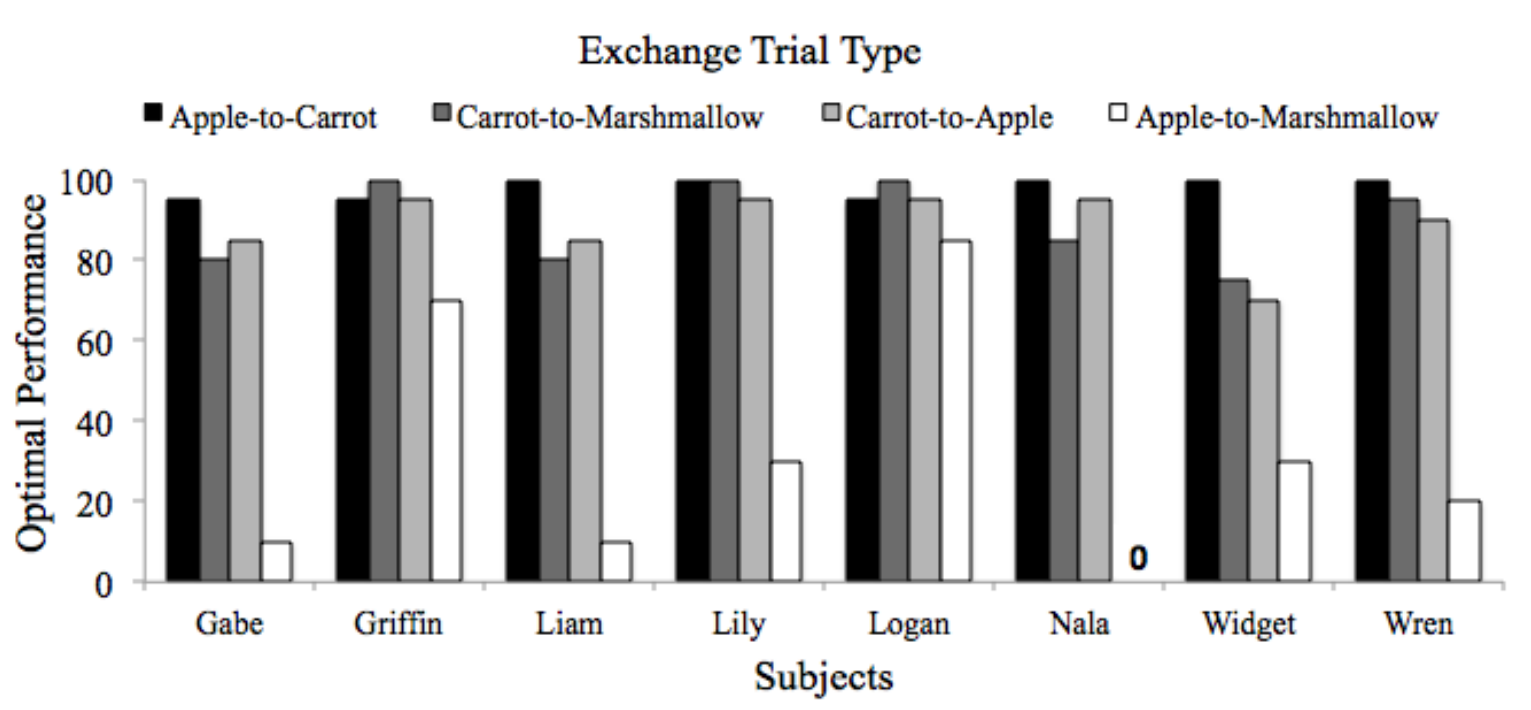

Figure 4. Exchange test performance across the four trial types in Experiment 1. Performance was collapsed across the two accumulation conditions (free and forced accumulation).

Accumulation $\mathbf{x}$ food exchange correlates. Finally, we correlated performance on the two tasks across all monkeys to determine whether a relationship existed between the separate but potentially related self-control measures. To do this, we used the percentage of total pellets earned across the five free accumulation sessions (maximum: 250, or 50 pellets per session) and the percentage of total optimal responses across the 10 food exchange sessions (maximum: 80, or 20 trials per trial type). There was not a significant correlation between performance on the two self-control tasks, $r(6)=.58, p=.133$.

\section{Discussion}

Although there was variability in the number of pellets earned by capuchin monkeys in the selfcontrol accumulation task and the number of higher-valued items obtained in the exchange task, in general there was good evidence of self-control in both tasks. The monkeys were trained on a novel exchange task that required inhibition of consuming the first food if a better food could be gained through exchange. The monkeys did not blindly exchange all food items, but typically exchanged when that behavior would lead to a better food reward (Quality Increasing trials) or did not exchange the food item if it was the better food reward available (Quality Decreasing trials). Exchanges that required foregoing a 
medium-valued food item (apple piece) or a higher-valued food item (mini-marshmallow) proved more difficult in both training and testing than an exchange of a lower-valued food item (carrot slice). Accumulation task performance varied widely, in which some monkeys (e.g., Lily and Logan) performed well across nearly all sessions of the experiment, accumulating nearly every food item, whereas other monkeys failed to accumulate more than a few pellets in any given session (e.g., Widget and Nala). These results in terms of variance across individuals are consistent with previous accumulation studies performed with these same subjects (e.g., Parrish et al., 2016).

With regards to self-control depletion effects, there were no differences in self-control performance in the food exchange task for any monkey as a function of the first task's requirement of inhibitory control. Regardless of whether the first task required self-control (free accumulation) or not (forced accumulation), monkey's performance in the food exchange task was consistent across these conditions, varying systematically as a function of the trial type rather than the depletion of self-control resources. These results, taken together with previous capuchin studies of the depletion hypothesis (De Petrillo et al., 2015) and the glucose hypothesis (Parrish et al., 2016), suggest that capuchin monkeys are not subject to self-control depletion effects which also has been reported in some human and comparative studies (e.g., Hagger \& Chatzisarantis, 2017; Hagger et al., 2010; Vadillo et al., 2016). Furthermore, monkeys' performance on the two self-control tasks failed to correlate, suggesting that these tasks perhaps are tapping into different mechanisms underlying self-control.

We next explored whether there were differential effects of depletion depending upon task order. Because all monkeys had more experience with the accumulation task from previous testing, it was possible that this task, although still clearly demanding given that most animals did not perform at ceiling, failed to deplete resources in the same manner in which a more novel and perhaps more challenging food exchange task would. In Experiment 2, we counterbalanced the presentation of the two self-control tasks, such that monkeys experienced the exchange task followed by the accumulation task on half of the sessions.

\section{Experiment 2}

\section{Method}

Subjects and apparatus. These were the same eight monkeys and apparatus as in Experiment 1.

\section{General Procedure}

Experiment 2 began within one week following the conclusion of Experiment 1. The monkeys completed the same two self-control tasks from Experiment 1 - the accumulation and food exchange tests. Here, we counterbalanced the order in which monkeys completed the two tasks to determine if there were differential depletion effects depending upon the self-control task completed first.

The accumulation task presented in the current experiment was identical to that of the free accumulation condition presented in Experiment 1 (pan unlocked with food contents accessible throughout the trial), thus requiring continual self-control by the monkey. We used the same delivery rate $(2 \mathrm{~s})$ and same food type (94-mg banana flavored pellets). Monkeys again could accumulate up to 50 items within a session.

The exchange task also was identical to that from Experiment 1 test phase. In order to test the greatest possible degree of depletion, we presented monkeys with the most difficult exchange trial type (Quality Increasing - apple-to-marshmallow) in which they needed to inhibit consuming a mediumvalued food and instead exchange that food for a higher-valued reward. Exchange trials were $30 \mathrm{~s}$ in length, regardless of exchange behavior. The exchange phase included eight total trials.

Each monkey completed 10 test sessions administered on different days. Each session included an accumulation phase with one free accumulation trial (with up to 50 pellets) and one exchange phase. Note that one monkey (Lily) accumulated a total of 53 pellets on one accumulation trial due to experimenter 
error. The total number of pellets was considered to be 50 for this trial, to keep the maximum trial count consistent.

There was a 6 min delay between the end of the first task and the start of the second task to allow time for task setup and for the animals to finish consuming their food items from the first task (the intertask interval for one session [for Griffin] was 3 min due to experimenter error).

Crucial to the current experimental design, in five of the 10 sessions, monkeys first completed the accumulation trial followed by the exchange trials. In the other five sessions, monkeys first completed the exchange trials followed by the accumulation trial. Across the 10 sessions, we alternated back and forth between the starting tasks, with accumulation followed by the exchange task in the first session (and vice versa for the second session and so on).

\section{Results}

Accumulation testing. Table 3 and Figure 5A present the accumulation test results. We reported the total number of pellets accumulated for each monkey across the five accumulation test days in which the accumulation task was presented first and across the five test days in which the accumulation task was presented second. We compared the number of pellets accumulated in each of these conditions (accumulation task first vs. accumulation task second) across all animals using a paired samples $t$-test. There was no significant difference in the total number of pellets accumulated per session when the task was presented before $(M=15.25, S D=14.35)$ or after $(M=18.38, S D=14.79)$ the exchange task, $t(39)=$ $-1.60, p=.118$. We also compared the number of pellets in the two conditions for each monkey. Consistent with the group analysis, there was no significant difference in pellet accumulation for seven out of eight monkeys, all $p s>.05$ ( $t$-test results presented in Table 3). The exception was Griffin, who accumulated significantly fewer pellets when the accumulation task was presented first $(M=9.20, S D=$ 7.66) than when the task was presented second $(M=17.80, S D=7.85), t(4)=-3.21, p=.032$. Importantly, this result is not consistent with the depletion hypothesis, which predicts a greater number of accumulated pellets when the accumulation task was presented first (prior to the exchange task).

Table 3

Accumulation Test Performance from Experiment 2 when the Task was Presented First (Prior to Exchange) Versus Second (After Exchange)

\begin{tabular}{lccc}
\hline & \multicolumn{2}{c}{ Total Pellets Accumulated (Task Maximum: 250) } & Task Order Analysis \\
Accumulation Task 1st & Accumulation Task 2nd & t-test Results \\
\hline Gabe & 58 & 86 & $t(4)=-1.61, p=.182$ \\
Griffin & 46 & 89 & $t(4)=-3.21, p=.032$ \\
Liam & 74 & 56 & $t(4)=2.21, p=.092$ \\
Lily & 174 & 165 & $t(4)=.14, p=.892$ \\
Logan & 151 & 169 & $t(4)=-.67, p=.539$ \\
Nala & 25 & 37 & $t(4)=-1.37, p=.242$ \\
Widget & 7 & 7 & $t(4)=--, p=--*$ \\
Wren & 75 & 126 & $t(4)=-1.48, p=.214$
\end{tabular}

Food exchange testing. Table 4 and Figure 5B present the exchange test results. We reported the total number of higher-valued items obtained for each monkey across the five exchange test days in which the exchange task was presented first and across the five test days in which the exchange task was 
presented second. In order to obtain the higher-valued food (mini-marshmallow), the monkey needed to inhibit consuming the relatively less-preferred food (apple piece) to trade with the experimenter.

(A) Accumulation

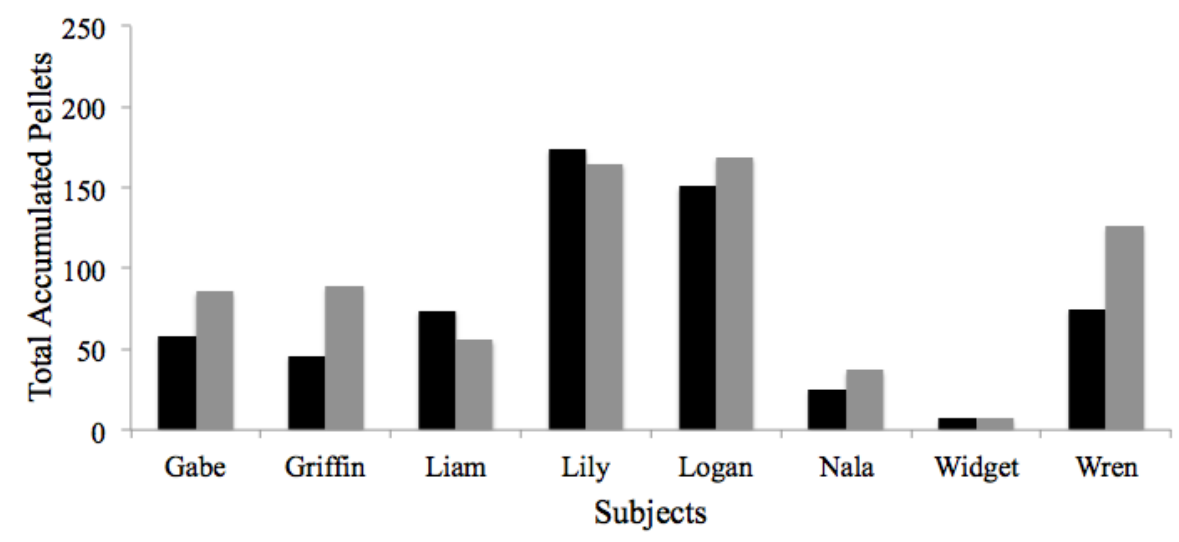

(B) Exchange

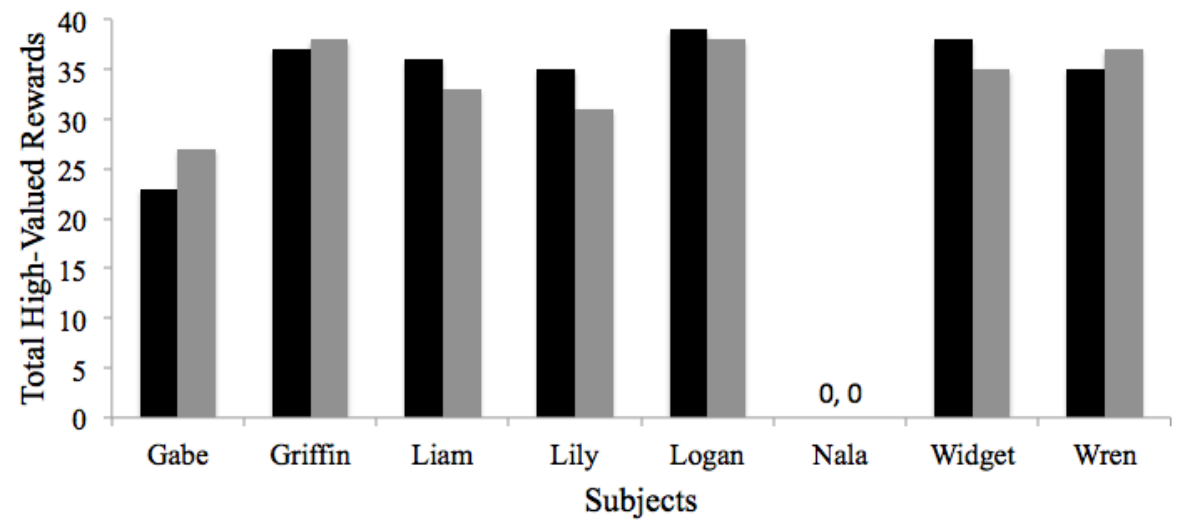

Figure 5. Experiment 2 test performance for the (A) accumulation self-control task, including the total number of pellets accumulated (maximum: 250 pellets) when the accumulation task was presented first (black bars) and when the accumulation task was presented second (gray bars). Test performance for the $(\mathbf{B})$ exchange self-control task, including the total number of higher-valued rewards obtained (maximum: 40 mini-marshmallows) when the exchange task was presented first (black bars) and when the exchange task was presented second (gray bars).

We compared the number of marshmallows received in each of these conditions (exchange task first vs. exchange task second) across all animals using a paired samples $t$-test. There was no significant difference in the number of marshmallows earned per session when the task was presented before $(M=$ $6.08, S D=2.92)$ instead of after $(M=5.98, S D=2.65)$ the accumulation task, $t(39)=0.36, p=.722$. We also compared the number of marshmallows received in the two conditions for each monkey across sessions using a paired samples $t$-test. Consistent with the group analysis, there was no significant difference in total marshmallows obtained for seven out of eight monkeys, all $p s>.05$ ( $t$-test results presented in Table 4). The exception was Lily, who obtained significantly more marshmallows when the exchange task was presented first $(M=7.00, S D=0.71)$ than when the task was presented second $(M=$ $6.20, S D=1.10), t(4)=4.00, p=.016$. This result was consistent with the depletion hypothesis, which predicts a greater number of higher-valued items obtained when the exchange task was presented first (prior to accumulation). 
Table 4

Exchange Test Performance from Experiment 2 when the Task was Presented First (Prior to Accumulation) Versus Second (After Accumulation)

\begin{tabular}{lccc}
\hline & $\begin{array}{c}\text { Total Mini-Marshmallows (Task Maximum: 40) } \\
\text { Exchange Task 1st }\end{array}$ & $\begin{array}{c}\text { Task Order Analysis } \\
\text { Exchange Task 2nd }\end{array}$ & \begin{tabular}{c}
$\boldsymbol{t}$-test Results \\
\hline Gabe
\end{tabular} $2^{-23}$ \\
Griffin & 37 & 27 & $t(4)=-0.39, p=.714$ \\
Liam & 36 & 38 & $t(4)=-0.41, p=.704$ \\
Lily & 35 & 33 & $t(4)=0.74, p=.501$ \\
Logan & 39 & 31 & $t(4)=4.00, p=.016$ \\
Nala & 0 & 38 & $t(4)=0.53, p=.621$ \\
Widget & 38 & 0 & $t(4)=--, p=--*$ \\
Wren & 35 & 35 & $t(4)=1.50, p=.208$ \\
\hline
\end{tabular}

$* t$ could not be calculated for Nala because the standard error of the difference was 0 .

\section{Relation of Accumulation and Food Exchange Performance}

We correlated overall performance by each monkey on the two self-control tasks. Despite a lack of a relationship between task performances in Experiment 1, we felt that it was beneficial to reassess this relationship given the extended experience with each task in the current experiment. Thus, we calculated the percentage of total pellets earned across all ten accumulation sessions, collapsing across task order (first vs. second; maximum pellets: 500, or 50 pellets per session). We also calculated the percentage of total higher-valued rewards obtained across the 10 food exchange sessions (maximum: 80, or 8 trials per session). As in Experiment 1, there was not a significant correlation between performance on the two selfcontrol tasks, $r(6)=.38, p=.35$.

\section{Discussion}

Consistent with Experiment 1, there was high variability in accumulation test performance by individual monkeys, with the same monkeys from Experiment 1 accumulating a relatively high number of pellets (e.g., Lily and Logan) in comparison to others who accumulated relatively few pellets (e.g., Widget and Nala). Similarly, there were individual differences in performance for the food exchange task with one monkey failing to exchange the apple piece for the marshmallow in any trials (Nala) whereas many other monkeys performed quite well on the exchange test in this experiment; see Table 4 and Figure 5B. As in Experiment 1, there was no correlation between the two self-control tasks for Experiment 2. Furthermore, there were no depletion effects observed among capuchin monkeys for either task order (accumulation followed by exchange or exchange followed by accumulation).

\section{General Discussion}

According to the ego-depletion model, self-control resources fatigue with exertion leaving subsequently fewer resources to devote to the next task (Baumeister, 2002). Recent comparative studies have added to this growing body of literature, with evidence consistent with the depletion effect among canines (Miller, 2013; Miller \& Bender, 2012; Miller et al., 2010, 2012) and also evidence to the contrary among more closely related primate species (De Petrillo et al., 2015; Parrish et al., 2016). This mixed evidence in the comparative literature mirrors recent meta-analyses using aggregate data from a variety of human adult ego-depletion tasks, suggesting that the story may in fact be more complicated than a single 
mechanism driving self-control (Hagger \& Chatzisarantis, 2017; Hagger et al., 2010; Vadillo et al., 2016). Using a consecutive task paradigm in the current study, monkeys were presented with the accumulation task and a food exchange task, both of which required sustained or continual self-control to inhibit eating a desirable food reward which facilitated the addition of or exchange for a better reward. Individual differences in task performance were observed, but no self-control depletion effects were seen in the monkeys' subsequent exchange performances in Experiment 1. Next, monkeys were presented with these tasks counterbalanced across individuals. No order effects were observed in the monkeys' performances in either self-control task in Experiment 2. Ultimately, we did not find evidence for the depletion effect in capuchin monkeys using the current consecutive task paradigm.

The two tasks selected to assess self-control in the current study are widely used in the comparative literature, produce interesting individual differences, and are influenced by a variety of predicted factors (e.g., food quality, quantity, visibility, food delivery rate, etc.). However, in the present study, performances in these two tasks appeared to be unrelated. Specifically, monkeys' exchange performance (calculated in terms of the total number of higher-valued rewards obtained) was not significantly correlated with accumulation performance (calculated as the total number of items accumulated) in either experiment. These results suggest that these tasks may not be related in terms of underlying mechanisms that support self-control performance, even though at face value both require inhibition of eating available food. These results are consistent with other human (Reynolds \& Schiffbauer, 2005) and comparative (Addessi et al., 2013) assessments of self-control, which also failed to demonstrate a correlation across self-control tasks. For example, Brucks et al. (2017a) recently tested 67 dogs on a battery of frequently-used inhibition and self-control tasks (e.g., middle cup test, delay of gratification, reversal learning) and reported no correlation of performance across tasks, suggesting that these tests likely measure different aspects of inhibitory-control and self-control. It is possible that depletion effects may emerge more readily in self-control tasks that correlate with one another and that rely on other forms of self-control, such as intertemporal choice responses.

Despite a lack of depletion effects in the current study, performance in the individual self-control tasks generated interesting data patterns independently of one another. Food exchange performance varied as a function of exchange trial type, such that some trials were objectively more difficult (in terms of selfcontrol) than others. For example, exchanges that required foregoing a medium-valued food item (apple piece to get a marshmallow) or a smaller quantity of the same food (one marshmallow to get three marshmallows) proved more difficult than an exchange of a lower-valued food item (carrot slice for an apple slice). Intuitively, this makes sense - it should be more difficult to inhibit consumption of a relatively more desired food type (apple or marshmallow) than a lower-valued item (carrot). Beyond differences in the qualitative exchanges, the monkeys never made a quantitative exchange (one marshmallow for three marshmallows). This pattern of results is consistent with past research that demonstrated quantitative exchanges are more difficult relative to qualitative exchanges for capuchin monkeys (Drapier, Chauvin, Dufour, Uhlrich, \& Thierry, 2005), chimpanzees (Beran et al., 2016), and corvids (Dufour, Wascher, Braun, Miller, \& Bugnyar, 2012; Wascher, Dufour, \& Bugnyar, 2012). Selfcontrol tasks that prove more difficult (e.g., quantitative exchanges) are important for future testing of depletion as they may utilize and subsequently deplete resources to a greater degree than objectively easier tasks (e.g., qualitative exchanges). Although the monkeys did not successfully exchange a lesser quantity for a greater quantity in the present study, we did not find evidence of depletion effects in Experiment 2 in which we exclusively presented the most difficult qualitative trial type from Experiment 1 (apple-to-marshmallow).

Accumulation task performance also varied widely across individual monkeys, a finding consistent with both current experiments and previous studies with these monkeys (Addessi et al., 2013; Evans et al., 2012; Paglieri et al., 2013; Parrish et al., 2016). We chose accumulation as our first task in Experiment 1 because self-control expenditure (measured as the number of pellets accumulated) is strictly dependent upon individual capability. We believed this design, by requiring inhibition, would be the best possible test of depletion effects in the capuchin monkey, a frenetic and highly active primate species. The use of a self-control task that required active engagement throughout as the first test, rather than a 
design in which animals are required to wait a pre-set amount of time or refrain from eating a specific type of food, differs from the typical design for consecutive self-control tasks. However, future studies that vary the initial task type might lead to different results for depletion studies among capuchin monkeys. For example, a task in which monkeys are required to engage in a self-control scenario but ultimately cannot obtain a food reward for their effort may be more taxing to their 'resources', leading to instances of self-control depletion.

The present results, coupled with recent attempts to capture self-control depletion and the glucose hypothesis among capuchin monkeys (De Petrillo et al., 2015; Parrish et al., 2016), suggest that nonhuman primates are not subject to decrements in self-control resources as a function of prior experience or expenditure. Furthermore, self-control performance across seemingly related tasks does not appear to correlate within individual monkeys in the present study. These results coupled with similar reports of a lack of correlation across self-control tasks (e.g., Addessi et al., 2013; Brucks et al., 2017a, Reynolds \& Schiffbauer, 2005) suggest that the mechanisms underlying inhibition and self-control are multifaceted. In the human literature, depletion effects are not firmly established and, when reported, may emerge due to other factors in addition to or aside from self-control resource depletion (e.g., boredom, fatigue, frustration, satiation, etc.). Comparative data would seem to argue against such depletion, at least when nonhuman primates are tested in ways that are comparable to the human tests that have sometimes reported evidence of depletion. Future studies of depletion effects among nonhuman species are necessary to rule out a variety of factors that may influence performance and task-to-task interference, including the type of testing paradigm used (consecutive task paradigm vs. concurrent task paradigm) and the nature of the stimuli presented (primary or secondary reinforcers). It remains possible that nonhuman primates (and other animals) may show evidence of depletion, but the present results suggest that depletion is not readily evident when back-to-back self-control tasks are completed, even when each task shows a range of capabilities across subjects. As such, and in line with the theme of this special issue, this is a meaningful failure to show consistency across self-control tasks in capuchin monkeys, and also in the failure to demonstrate depletion effects in monkeys despite the use of well-established tasks that rely on self-control and delay of gratification.

\section{Acknowledgments}

Support for this research was provided by NICHD (grant HD-060563) and NSF (grant BCS 1552405). We thank the animal care and enrichment staff at the Language Research Center for maintaining the health and wellbeing of the primates and making this research possible.

\section{References}

Ackerman, J. M., Goldstein, N. J., Shapiro, J. R., \& Bargh, J. A. (2011). You wear me out: The vicarious depletion of self-control. Psychological Science, 20, 326-332.

Addessi, E., Paglieri, F., Beran, M. J., Evans, T. A., Macchitella, L., ...Focaroli, V. (2013). Delay choice versus delay maintenance: Different measures of delayed gratification in capuchin monkeys (Cebus apella). Journal of Comparative Psychology, 127, 392-398.

Addessi, E., \& Rossi, S. (2011). Tokens improve capuchin performance in the reverse-reward contingency task. Proceedings of the Royal Society of London, Series B, 278, 849-854.

Ainslie, G. W. (1974). Impulse control in pigeons. Journal of the Experimental Analysis of Behavior, 21, $485-489$.

Anderson, J. R., Kuroshima, H., \& Fujita, K. (2010). Delay of gratification in capuchin monkeys (Cebus apella) and squirrel monkeys (Saimiri sciureus). Journal of Comparative Psychology, 124, 205-210.

Baumeister, R. F. (2002). Yielding to temptation: Self-control failure, impulsive purchasing, and consumer behavior. Journal of Consumer Research, 28, 670-676.

Baumeister, R. F., Bratslavsky, E., Muraven, M., \& Tice, D. M. (1998). Ego depletion: Is the active self a limited resource? Journal of Personality and Social Psychology, 74, 1252-1265.

Baumeister, R. F., Heatherton, T. F., \& Tice, D. M. (1994). Losing control: How and why people fail at selfregulation. San Diego, CA: Academic Press. 
Baumeister, R. F., Sparks, E. A., Stillman, T. F., \& Vohs, K. D. (2008). Free will in consumer behavior: Selfcontrol, ego depletion, and choice. Journal of Consumer Psychology, 18, 4-13.

Baumeister, R. F., Vohs, K. D., \& Tice, D. M. (2007). The strength model of self-control. Current Directions in Psychological Science, 16, 351-355.

Beran, M. J., (2002). Maintenance of self-imposed delay of gratification by four chimpanzees (Pan troglodytes) and an orangutan (Pongo pygmaeus). Journal of General Psychology, 129, 49-66.

Beran, M. J. (2015). The comparative science of "self-control": What are we talking about? Frontiers in Psychology, $6,51$.

Beran, M. J., \& Evans, T. A., (2006). Maintenance of delay of gratification by four chimpanzees (Pan troglodytes): The effects of delayed reward visibility, experimenter presence, and extended delay intervals. Behavioural Processes, 73, 315-324.

Beran, M. J., \& Evans, T. A. (2012). Language-trained chimpanzees (Pan troglodytes) delay gratification by choosing token exchange over immediate reward consumption. American Journal of Primatology, 74, 864870.

Beran, M. J., Perdue, B. M., Rossettie, M. S., James, B. T., Whitham, W., Walker, B., ...Parrish, A. E. (2016). Selfcontrol assessments of capuchin monkeys with the rotating tray task and the accumulation task. Behavioural Processes, 129, 68-79.

Beran, M. J., Rossettie, M. S., \& Parrish, A. E. (2016). Trading up: Chimpanzees (Pan troglodytes) show selfcontrol through their exchange behavior. Animal Cognition, 19, 109-121.

Beran, M. J., Savage-Rumbaugh, E. S., Pate, J. L., \& Rumbaugh, D. M. (1999). Delay of gratification in chimpanzees (Pan troglodytes). Developmental Psychobiology, 34, 119-127.

Berns, G. S., Laibson, D., \& Loewenstein, G. (2007). Intertemporal choice-toward an integrative framework. Trends in Cognitive Sciences, 11, 482-488.

Blanchard, T. C., \& Hayden, B. Y. (2015). Monkeys are more patient in a foraging task than in a standard intertemporal choice task. PloS one, 10, e0117057.

Bourjade, M., Thierry, B., Call, J., \& Dufour, V. (2012). Are monkeys able to plan for future exchange? Animal Cognition, 15, 783-795.

Bramlett, J. L., Perdue, B. M., Evans, T. A., \& Beran, M. J. (2012). Capuchin monkeys (Cebus apella) let lesser rewards pass them by to get better rewards. Animal Cognition, 15, 963-969.

Brucks, D., Marshall-Pescini, S., Wallis, L. J., Huber, L., \& Range, F. (2017a). Measures of dogs' inhibitory control abilities do not correlate across tasks. Frontiers in Psychology, 8, 849

Brucks, D., Soliani, M., Range, F., \& Marshall-Pescini, S. (2017b). Reward type and behavioural patterns predict dogs' success in a delay of gratification paradigm. Scientific Reports, 7, 42459.

Carter, E. C., \& McCullough, M. E. (2014). Publication bias and the limited strength model of self-control: Has the evidence for ego depletion been overestimated? Frontiers in Psychology, 5, 823.

Deluty, M. Z. (1978). Self-control and impulsiveness involving aversive events. Journal of Experimental Psychology: Animal Behavior Processes, 4, 250-266.

De Petrillo, F., Micucci, A., Gori, E., Truppa, V., Ariely, D., \& Addessi, E. (2015). Self-control depletion in tufted capuchin monkeys (Sapajus spp.): Does delay of gratification rely on a limited resource? Frontiers in Psychology, 6, 1193.

Drapier, M., Chauvin, C., Dufour, V., Uhlrich, P., \& Thierry, B. (2005). Food-exchange with humans in brown capuchin monkeys. Primates, 46, 241-248.

Dufour, V., Pelé, M., Sterck, E. H. M., \& Thierry, B. (2007). Chimpanzee (Pan troglodytes) anticipation of food return: Coping with waiting time in an exchange task. Journal of Comparative Psychology, 121, 145-155.

Dufour, V., Wascher, C. A. F., Braun, A., Miller, R., \& Bugnyar, T. (2012). Corvids can decide if a future exchange is worth waiting for. Biology Letters, 8, 201-204.

Egan, P. M., Hirt, E. R., \& Karpen, S. C. (2011). Taking a fresh perspective: Vicarious restoration as a means of recovering self-control. Journal of Experimental Social Psychology, 48, 457-465.

Evans, T. A., \& Beran, M. J. (2007). Delay of gratification and delay maintenance by rhesus macaques (Macaca mulatta). Journal of General Psychology, 134, 199-216.

Evans, T. A., \& Beran, M. J. (2014). Monkeys wait to begin a computer task when waiting makes their responses more effective. Animal Behavior and Cognition, 1, 36-50.

Evans, T. A., Beran, M. J., Paglieri, F., \& Addessi, E. (2012). Delaying gratification for food and tokens in capuchin monkeys (Cebus apella) and chimpanzees (Pan troglodytes): When quantity is salient, symbolic stimuli do not improve performance. Animal Cognition, 15, 539-548. 
Evans, T. A., Perdue, B. M., Parrish, A. E., \& Beran, M. J. (2014). Working and waiting for better rewards: Selfcontrol in two monkey species (Cebus apella and Macaca mulatta). Behavioural Processes, 103, 236-242.

Evans, T. A., \& Westergaard, G. C. (2006). Self-control and tool use in tufted capuchin monkeys (Cebus apella). Journal of Comparative Psychology, 120, 163-166.

Gailliot, M. T., \& Baumeister, R. F. (2007). The physiology of willpower: Linking blood glucose to self-control. Personality and Social Psychology Review, 11, 303-327.

Green, L., Myerson, J., Holt, D. D., Slevin, J. R., \& Estle, S. J. (2004). Discounting of delayed food rewards in pigeons and rats: Is there a magnitude effect? Journal of the Experimental Analysis of Behavior, 81, 39-50.

Grosch, J., \& Neuringer, A. (1981). Self-control in pigeons under the Mischel paradigm. Journal of the Experimental Analysis of Behavior, 35, 3-21.

Hackenberg, T. D., \& Vaidya, M. (2003). Determinants of pigeons choices in token-based self-control procedures. Journal of the Experimental Analysis of Behavior, 79, 207-218.

Hagger, M. S., \& Chatzisarantis, N. L. D. (2017). A multilab preregistered replication of the ego-depletion effect. Perspectives on Psychological Science, 11, 546-573.

Hagger, M. S., Wood, C., Stiff, C., \& Chatzisarantis, N. L. D. (2010). Ego depletion and the strength model of selfcontrol: A meta-analysis. Psychological Bulletin, 136, 495-525.

Hillemann, F., Bugnyar, T., Kotrschal, K., \& Wascher, C. A. (2014). Waiting for better, not for more: Corvids respond to quality in two delay maintenance tasks. Animal Behaviour, 90, 1-10.

Inzlicht, M., \& Schmeichel, B. J. (2012). What is ego depletion? Toward a mechanistic revision of the resource model of self-control. Perspectives on Psychological Science, 7, 450-463.

Inzlicht, M., Schmeichel, B. J., \& Macrae, C. N. (2014). Why self-control seems (but may not be) limited. Trends in Cognitive Sciences, 18, 127-133.

Job, V., Walton, G. M., Bernecker, K., \& Dweck, C. S. (2013). Beliefs about willpower determine the impact of glucose on self-control. Proceedings of the National Academy of Sciences, 110, 14837-14842.

Judge, P. G., \& Essler, J. (2013). Capuchin monkeys exercise self-control by choosing token exchange over an immediate reward. International Journal of Comparative Psychology, 26, 256-266.

Koepke, A. E., Gray, S. L., \& Pepperberg, I. M. (2015). Delayed gratification: A grey parrot (Psittacus erithacus) will wait for a better reward. Journal of Comparative Psychology, 129, 339-346.

Lange, F., \& Eggert, F. (2014). Sweet delusion. Glucose drinks fail to counteract ego depletion. Appetite, 75, 54-63.

Logue, A. W. (1988). Research on self-control: An integrating framework. Behavioral and Brain Sciences, 11, 665679.

Logue, A. W., \& Chavarro, A. (1987). Effect on choice of absolute and relative values of reinforcer delay, amount, and frequency. Journal of Experimental Psychology: Animal Behavior Processes, 13, 280-291.

MacLean, E. L., Hare, B., Nunn, C. L., Addessi, E., Amici, F., Anderson, R. C., ...Boogert, N. J. (2014). The evolution of self-control. Proceedings of the National Academy of Sciences, 111, E2140-E2148.

Mayack, C., \& Naug, D. (2015). Starving honeybees lose self-control. Biology Letters, 11, 20140820.

Miller, H. C. (2013). The role of glucose on executive control vigor: Evidence in support of comparative research. International Journal of Comparative Psychology, 26, 208-220.

Miller, H. C., \& Bender, C. (2012). The breakfast effect: Dogs (Canis familiaris) search more accurately when they are less hungry. Behavioural Processes, 91, 313-317.

Miller, H. C., DeWall, C. N., Pattison, K., Molet, M., \& Zentall, T. R. (2012). Too dog tired to avoid danger: Selfcontrol depletion in canines increases behavioral approach toward an aggressive threat. Psychonomic Bulletin \& Review, 19, 535-540.

Miller, H. C., Pattison, K. F., DeWall, C. N., Rayburn-Reeves, R., \& Zentall, T. R. (2010). Self-control without a "self'? Common self-control processes in humans and dogs. Psychological Science, 21, 534-538.

Mischel, W. (1974). Processes in delay of gratification. In L. Berkowitz (Ed.), Advances in experimental social psychology (pp. 249-292). New York: Academic Press.

Mischel, W. (2014). The marshmallow test: Mastering self-control. New York: Hachette Book Group.

Muraven, M. (2010). Building self-control strength: Practicing self-control leads to improved self-control performance. Journal of Personality and Social Psychology, 46, 465-468.

Muraven, M., Tice, D. M., \& Baumeister, R. F. (1998). Self-control as limited resource: Regulatory depletion patterns. Journal of Personality and Social Psychology, 74, 774-789.

Navarick, D. J., \& Fantino, E. (1976). Self-control and general models of choice. Journal of Experimental Psychology: Animal Behavior Processes, 2, 75-87. 
Paglieri, F., Focaroli, V., Bramlett, J., Tierno, V., McIntyre, J. M., Addessi, E., ...Beran, M. J. (2013). The hybrid delay task: Can capuchin monkeys (Cebus apella) sustain a delay after an initial choice to do so? Behavioural Processes, 94, 45-54.

Parrish, A. E., Emerson, I.D., Rossettie, M. S., \& Beran, M. J. (2016). Testing the glucose hypothesis among capuchin monkeys: Does glucose boost self-control? Behavioral Sciences, 6, 16.

Parrish, A. E., Evans, T. E., Perdue, B. M., \& Beran, M. J. (2013). Chimpanzees (Pan troglodytes) transfer tokens repeatedly with a partner to accumulate rewards in a self-control task. Animal Cognition, 16, 627-636.

Parrish, A. E., Perdue, B. M., Stromberg, E. E., Bania, A. E., Evans, T. A., \& Beran, M. J., (2014). Delay of gratification by orangutans (Pongo pygmaeus) in the accumulation task. Journal of Comparative Psychology, 128, 209-214.

Pelé, M., Dufour, V., Micheletta, J., \& Thierry, B. (2010). Long-tailed macaques display unexpected waiting abilities in exchange tasks. Animal Cognition, 13, 263-271.

Pelé, M., Micheletta, J., Uhlrich, P., Thierry, B., \& Dufour, V. (2011). Delay maintenance in tonkean macaques (Macaca tonkeana) and brown capuchin monkeys (Cebus apella). International Journal of Primatology, 32, 149-166.

Rachlin, H. (2009). The science of self-control. Cambridge, MA: Harvard University Press.

Rachlin, H., \& Green, L. (1972). Commitment, choice and self-control. Journal of the Experimental Analysis of Behavior, 17, 15-22.

Ramseyer, A., Pelé, M., Dufour, V., Chauvin, C., \& Thierry, B. (2006). Accepting loss: The temporal limits of reciprocity in brown capuchin monkeys. Proceedings of the Royal Society of London B: Biological Sciences, 273, 179-184.

Reynolds, B., \& Schiffbauer, R. (2005). Delay of gratification and delay discounting: A unifying feedback model of delay-related impulsive behavior. The Psychological Record, 55, 439-460.

Sanders, M. A., Shirk, S. D., Burgin, C. J., \& Martin, L. L. (2012). The gargle effect: Rinsing the mouth with glucose enhances self-control. Psychological Science, 23, 1470-1472.

Schmeichel, B. J., Vohs, K. D., \& Baumeister, R. F. (2003). Ego depletion and intelligent performance: Role of the self in logical reasoning and other information processing. Journal of Personality and Social Psychology, $85,33-46$.

Shoda, Y., Mischel, W., \& Peake, P. K. (1990). Predicting adolescent cognitive and self-regulatory competencies from preschool delay of gratification: Identifying diagnostic conditions. Developmental Psychology, 26, 978-986.

Stevens, J. R., Hallinan, E. V., \& Hauser, M. D. (2005). The ecology and evolution of patience in two New World monkeys. Biology Letters, 1, 223-226.

Stevens, J. R., \& Mühlhoff, N. (2012). Intertemporal choice in lemurs. Behavioural Processes, 89, 121-127.

Stevens, J. R., Rosati, A. G., Heilbronner, S. R., \& Mühlhoff, N. (2011). Waiting for grapes: Expectancy and delayed gratification in bonobos. International Journal of Comparative Psychology, 24, 99-111.

Tobin, H., Chelonis, J. J., \& Logue, A. W. (1993). Choice in self-control paradigms using rats. The Psychological Record, 43, 441-454.

Vadillo, M. A., Gold, N., \& Osman, M. (2016). The bitter truth about sugar and willpower: The limited evidential value of the glucose model of ego depletion. Psychological Science, 27, 1207-1214.

van Haaren, F., van Hest, A., \& van de Poll, N. E. (1988). Self-control in male and female rats. Journal of the Experimental Analysis of Behavior, 49, 201-211.

Vick, S. J., Bovet, D., \& Anderson, J. R. (2010). How do African grey parrots (Psittacus erithacus) perform on a delay of gratification task? Animal Cognition, 13, 351-358.

Vohs, K. D., Baumeister, R. F., Schmeichel, B. J., Twenge, J. M., Nelson, N. M., \& Tice, D. M. (2008). Making choices impairs subsequent self-control: A limited-resource account of decision making, self-regulation, and active initiative. Journal of Personality and Social Psychology, 94, 883-898.

Wascher, C. A., Dufour, V., \& Bugnyar, T. (2012). Carrion crows cannot overcome impulsive choice in a quantitative exchange task. Frontiers in Psychology, 3. 